\title{
Beneficial Prognostic Effects of Statins in Heart Failure With Preserved Ejection Fraction (HFpEF) Patients - HFpEF as a Manifestation of Systemic Disease -
}

\author{
Tatsuo Aoki, MD, PhD
}

$\mathbf{E}$ pidemiologic studies indicate that almost half of heart failure (HF) patients have preserved ejection fraction and that this proportion has increased over time. ${ }^{1,2}$ Patients with HF with preserved ejection fraction (HFpEF) have better prognosis and are more likely to die from non-cardiovascular causes than those with HF with reduced ejection fraction (HFrEF). ${ }^{3}$ Also, standard phar-

\section{Article p 357}

macological treatments for HFrEF including angiotensinconverting enzyme inhibitors, angiotensin receptor blockers, spironolactone and $\beta$-blocker have failed to improve the prognosis of HFpEF. ${ }^{4}$ Thus, patients with HFpEF have

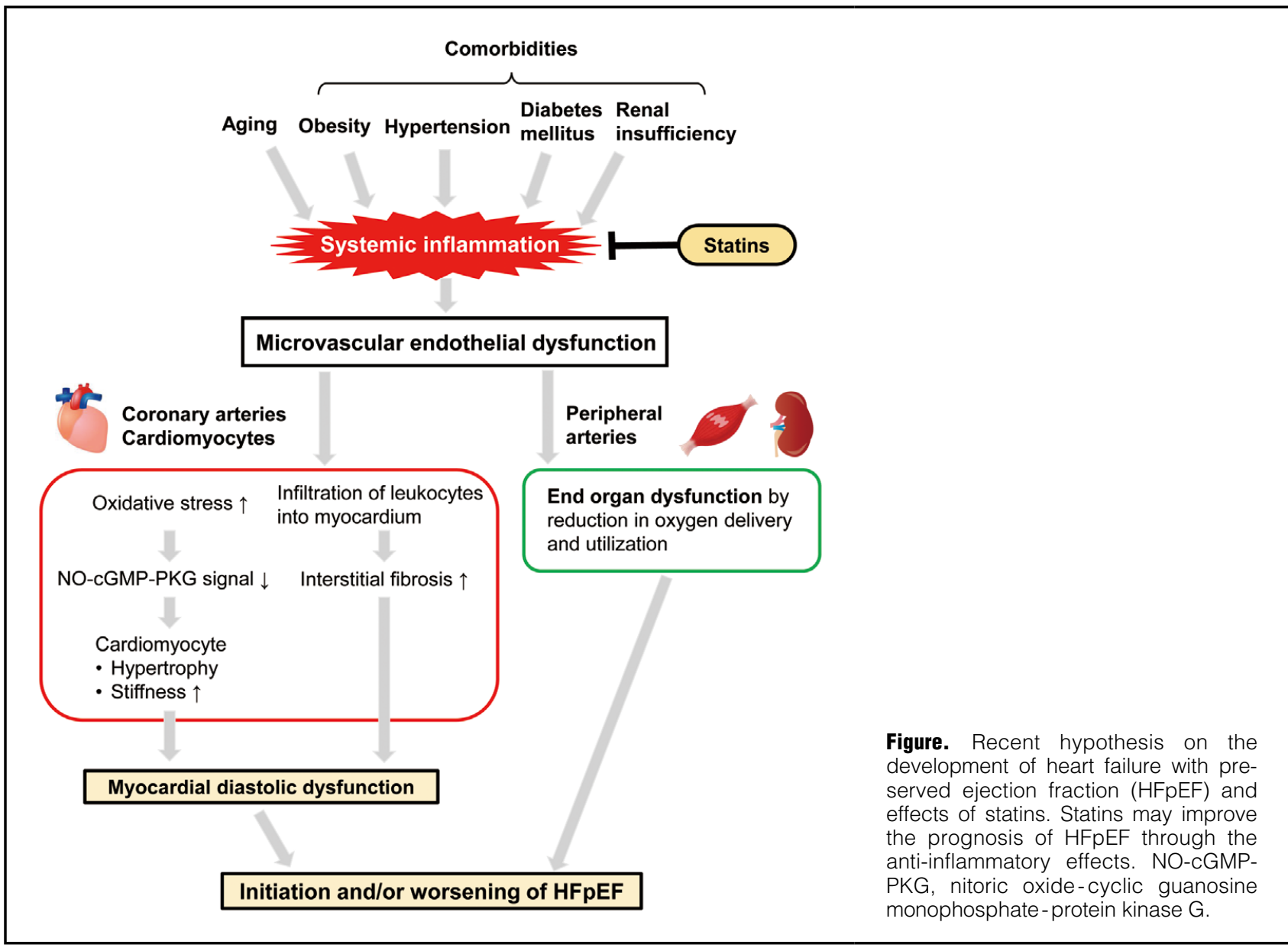

The opinions expressed in this article are not necessarily those of the editors or of the Japanese Circulation Society.

Received November 26, 2018; accepted November 26, 2018; J-STAGE Advance Publication released online December 11, 2018

Department of Cardiovascular Medicine, Tohoku University Graduate School of Medicine, Sendai, Japan

Mailing address: Tatsuo Aoki, MD, PhD, Department of Cardiovascular Medicine, Tohoku University Graduate School of Medicine, Seiryo-machi, Aoba-ku, Sendai 980-8575, Japan. E-mail: t.aoki@cardio.med.tohoku.ac.jp

ISSN-1346-9843 All rights are reserved to the Japanese Circulation Society. For permissions, please e-mail: cj@j-circ.or.jp 
different clinical features from those with HFrEF, associated with the different pathophysiology of HF. The pathophysiology of HFpEF remains incompletely defined, but traditionally it has been attributed to hypertensive left ventricular (LV) remodeling. Recent studies suggested that the systemic inflammation associated with aging, and comorbidities such as obesity, hypertension, diabetes mellitus, and renal insufficiency, are involved in the pathogenesis of HFpEF.,5 Although inflammatory biomarkers, such as soluble interleukin-1 receptor-like 1, C-reactive protein, and growth differentiation factor-15, are elevated in both types of $\mathrm{HF},{ }^{6,7}$ the extent of inflammation is higher in $\mathrm{HFpEF}$ than in $\mathrm{HFrEF}{ }^{6}$ suggesting that systemic inflammation plays a more important role in HFpEF than in HFrEF. Systemic inflammation in HFpEF may accelerate myocardial remodeling and dysfunction through coronary microvascular endothelial dysfunction, ${ }^{8}$ with subsequent myocardial infiltration of activated leukocytes and interstitial fibrosis without cardiomyocyte death. ${ }^{\mathbf{8}, 9}$ These conditions also cause oxidative stress that inhibits the nitric oxide-cyclic guanosine monophosphate-protein kinase $G$ signaling pathway, resulting in cardiomyocyte hypertrophy and enhanced myofiber stiffness. ${ }^{10}$ Thus, unlike HFrEF, in which remodeling is driven by cardiomyocyte cell death due to ischemia, infection, or toxicity, the central pathophysiology of HFpEF involves myocardial remodeling with limited cardiomyocyte cell damage and systemic endothelial dysfunction associated with systemic inflammation. 5 Thus, statins with anti-inflammatory effects could improve the prognosis of HFpEF (Figure).

The prognostic impact of statins in patients with $\mathrm{HF}$ is controversial. A large-scale landmark trial, CORONA, failed to demonstrate the prognostic benefits of statins in HF patients. ${ }^{11}$ Given, however, the subjects were limited to HFrEF patients, the prognostic impact of statins on $\mathrm{HFpEF}$ remains to be elucidated. ${ }^{11}$ In this issue of the Journal, Marume et al report that the use of statins is associated with reduced all-cause mortality in HFpEF patients, regardless of serum cholesterol level in the JASPER Study.12 Although previous studies have already demonstrated that the use of statins is associated with improved all-cause mortality in HFpEF patients, ${ }^{13,14}$ this study examined whether the use of statins is associated with better prognosis in HFpEF patients, particularly in those without coronary artery disease (CAD). Nochioka et al also reported in their Chart-2 Study that the use of statins was associated with better prognosis of HFpEF, regardless of the presence or absence of CAD. ${ }^{13}$ In the JASPER Study, the statin use was also associated with reduced all-cause mortality and hospitalization due to worsening of HF, but was not associated with cardiovascular death. In animal models, statins improve endothelial redox balance and restore nitric oxide bioavailability independently of low-density lipoprotein, ${ }^{15}$ attenuate oxidative stress, prevent progression of cardiac hypertrophy and extra-cellular remodeling, and subsequently ameliorate LV function. ${ }^{16,17}$ These findings support the positive outcomes associated with statins in HFpEF patients. In $\mathrm{HFpEF}$ patients, microvascular endothelial dysfunction develops not only in coronary arteries but also in systemic arteries, leading to initiation and worsening of HFpEF through myocardial diastolic dysfunction and end-organ dysfunction (Figure). Thus, HFpEF could be regarded as a manifestation of "systemic disease", and thus statins could improve the prognosis of HFpEF through improvement of systemic endothelial and end-organ func- tions. As noted here, several experimental studies have indicated the beneficial effects of statins. The mechanism of the possible beneficial prognostic effects of statins, however, remains to be elucidated, which was also the case in the Marume et al study. ${ }^{\mathbf{1 2}}$ Thus, further studies are needed to confirm the beneficial prognostic effects of statins in HFpEF patients.

\section{Disclosures}

The author declares no conflicts of interest.

\section{References}

1. Owan T, Hodge D, Herges R, Jacobsen S, Roger V, Redfield M. Trends in prevalence and outcome of heart failure with preserved ejection fraction. $N$ Engl J Med 2006; 355: 251 -259.

2. Tsuji K, Sakata Y, Nochioka K, Miura M, Yamauchi T, Onose $\mathrm{T}$, et al. Characterization of heart failure patients with mid-range left ventricular ejection fraction: A report from the CHART-2 Study. Eur J Heart Fail 2017; 19: 1258-1269.

3. Chan MM, Lam CS. How do patients with heart failure with preserved ejection fraction die? Eur J Heart Fail 2013; 15: 604-613.

4. Redfield MM. Heart failure with preserved ejection fraction. $N$ Engl J Med 2016; 375: 1868 - 1877.

5. Shah SJ, Kitzman DW, Borlaug BA, van Heerebeek L, Zile MR, Kass DA, et al. Phenotype-specific treatment of heart failure with preserved ejection fraction: A multiorgan roadmap. Circulation 2016; 134: 73-90.

6. Sanders-van Wijk S, van Empel V, Davarzani N, Maeder MT, Handschin R, Pfisterer ME, et al. Circulating biomarkers of distinct pathophysiological pathways in heart failure with preserved vs. reduced left ventricular ejection fraction. Eur J Heart Fail 2015; 17: 1006-1014.

7. Santhanakrishnan R, Chong JP, Ng TP, Ling LH, Sim D, Leong KT, et al. Growth differentiation factor 15, ST2, high-sensitivity troponin $\mathrm{T}$, and $\mathrm{N}$-terminal pro brain natriuretic peptide in heart failure with preserved vs. reduced ejection fraction. Eur J Heart Fail 2012; 14: 1338-1347.

8. Franssen C, Chen S, Unger A, Korkmaz HI, De Keulenaer GW, Tschöpe C, et al. Myocardial microvascular inflammatory endothelial activation in heart failure with preserved ejection fraction. JACC Heart Fail 2016; 4: 312-324.

9. Westermann D, Lindner D, Kasner M, Zietsch C, Savvatis K, Escher F, et al. Cardiac inflammation contributes to changes in the extracellular matrix in patients with heart failure and normal ejection fraction. Circ Heart Fail 2011; 4: 44-52.

10. van Heerebeek L, Hamdani N, Falcão-Pires I, Leite-Moreira AF, Begieneman MP, Bronzwaer JG, et al. Low myocardial protein kinase $\mathrm{G}$ activity in heart failure with preserved ejection fraction. Circulation 2012; 126: 830-839.

11. Kjekshus J, Apetrei E, Barrios V, Böhm M, Cleland JG, Cornel $\mathrm{JH}$, et al. Rosuvastatin in older patients with systolic heart failure. N Engl J Med 2007; 357: 2248-2261.

12. Marume K, Takashio S, Nagai T, Tsujita K, Saito Y, Yoshikawa $\mathrm{T}$, et al. Effect of statins on mortality in heart failure with preserved ejection fraction without coronary artery disease: Report from the JASPER Study. Circ J 2019; 83: 357-367.

13. Nochioka K, Sakata Y, Miyata S, Miura M, Takada T, Tadaki $\mathrm{S}$, et al. Prognostic impact of statin use in patients with heart failure and preserved ejection fraction. Circ J 2015; 79: 574-582.

14. Oikawa T, Sakata Y, Nochioka K, Miura M, Tsuji K, Onose T, et al. Prognostic impact of statin intensity in heart failure patients with ischemic heart disease: A report from the CHART-2 Study. $J$ Am Heart Assoc 2018; 7: e007524.

15. Antoniades C, Bakogiannis C, Leeson P, Guzik TJ, Zhang MH, Tousoulis D, et al. Rapid, direct effects of statin treatment on arterial redox state and nitric oxide bioavailability in human atherosclerosis via tetrahydrobiopterin-mediated endothelial nitric oxide synthase coupling. Circulation 2011; 124: 335-345.

16. Takemoto $\mathrm{M}$, Node $\mathrm{K}$, Nakagami H, Liao Y, Grimm M, Takemoto Y, et al. Statins as antioxidant therapy for preventing cardiac myocyte hypertrophy. J Clin Invest 2001; 108: 1429-1437.

17. Ichihara S, Noda A, Nagata K, Obata K, Xu J, Ichihara G, et al. Pravastatin increases survival and suppresses an increase in myocardial matrix metalloproteinase activity in a rat model of heart failure. Cardiovasc Res 2006; 69: 726-735. 